\title{
Upper Gastrointestinal Mucosal Injury and Symptoms in Elderly Low-Dose Aspirin Users
}

\author{
Yuji Shimada, Akihito Nagahara, Mariko Hojo, Daisuke Asaoka, Hitoshi Sasaki, \\ Hiroya Ueyama, Kenshi Matsumoto, and Sumio Watanabe \\ Department of Gastroenterology, Juntendo University School of Medicine, 2-1-1 Hongo Bunkyo-ku, Tokyo 113-8421, Japan \\ Correspondence should be addressed to Yuji Shimada; yshimada@juntendo.ac.jp
}

Received 17 November 2014; Revised 27 December 2014; Accepted 29 December 2014

Academic Editor: Peter J. Whorwell

Copyright (c) 2015 Yuji Shimada et al. This is an open access article distributed under the Creative Commons Attribution License, which permits unrestricted use, distribution, and reproduction in any medium, provided the original work is properly cited.

\begin{abstract}
Background. We investigated the prevalence, symptoms, and QOL impact of esophageal (EI), gastric (GI), and duodenal mucosal injury (DI) individually between low-dose aspirin (LDA) users and nonusers to reveal the clinical features of LDA-related mucosal injury. Methods. Data were extracted from the records of subjects who underwent upper gastrointestinal endoscopy at our department between April 2008 and December 2013. Responses from 3162 elderly patients on Frequency Scale for Symptoms of GERD (FSSG) and SF-8 QOL questionnaires (SF-8) were analyzed. FSSG items were classified into total score (TS), reflux score (RS), and dyspepsia score (DS). The SF-8 questionnaire consisted of the physical component summary (PCS) and mental component summary (MCS). Results. Prevalence among LDA users and nonusers, respectively, was $9.6 \%$ and $10.0 \%(P=0.83)$ for EI, 35.9\% and $27.5 \%(P=0.0027)$ for GI, 3.3\% and 3.4\% $(P=0.84)$ for DI, and $8.2 \%$ and $5.2 \%(P=0.036)$ for mucosal injury in 2 or more organs. LDA users diagnosed with EI had significantly lower PCS, LDA users diagnosed with GI had significantly lower DS, and LDA users diagnosed with DI had significantly lower RS and significantly lower MCS. Conclusion. These results provide important clinical information indicating that symptom-based management is not appropriate in LDA users regarding upper gastrointestinal mucosal injury.
\end{abstract}

\section{Introduction}

Low-dose aspirin (LDA) plays an important role in the prevention of atherosclerosis-related diseases through its antiplatelet effects $[1,2]$. LDA use is increasing dramatically around the world $[1,3]$. In Japan in particular, the number of LDA users is expected to increase due to the country's aging population [4]. However, it is known that in clinical practice LDA causes upper gastrointestinal mucosal damage, which can lead to critical conditions related to ulcer bleeding [5]. Additionally, among elderly patients, mucosal injury can easily develop into serious ulceration and bleeding, asymptomatically [6]. Therefore, it is important to determine precisely where and how frequently mucosal injury occurs in elderly patients taking LDA. For this reason, the current study focused exclusively on elderly patients ( $>65$ years of age).

There have been many reports about LDA-related gastrointestinal complications; however, most of them investigated the relationship between LDA and gastrointestinal bleeding or bleeding risk [7-10]. Other studies have simply focused on the relationship between LDA and upper gastrointestinal mucosal injury, but most did not investigate upper gastrointestinal mucosal injury in individual organs (i.e., esophagus, stomach, and duodenum). We felt that it was important to understand the features of LDA-related mucosal injury, specifically in individual organs, because it would be helpful in identifying the site of mucosal injury in patients taking LDA. Moreover, few studies have investigated subjects diagnosed with mucosal injury in 2 or more organs [11-14].

We also investigated the clinical features of patients with LDA-related upper gastrointestinal mucosal injury based on symptomatology at the time of endoscopy as assessed using questionnaires. It has been documented that continuous LDA treatment leads to worsening of various upper gastrointestinal symptoms [15-17]. Conversely, 1 study reported that there were no significant differences between ulcer and nonulcer patients taking LDA with respect to the frequency and severity of symptoms [11]. Additionally, no studies to date have 


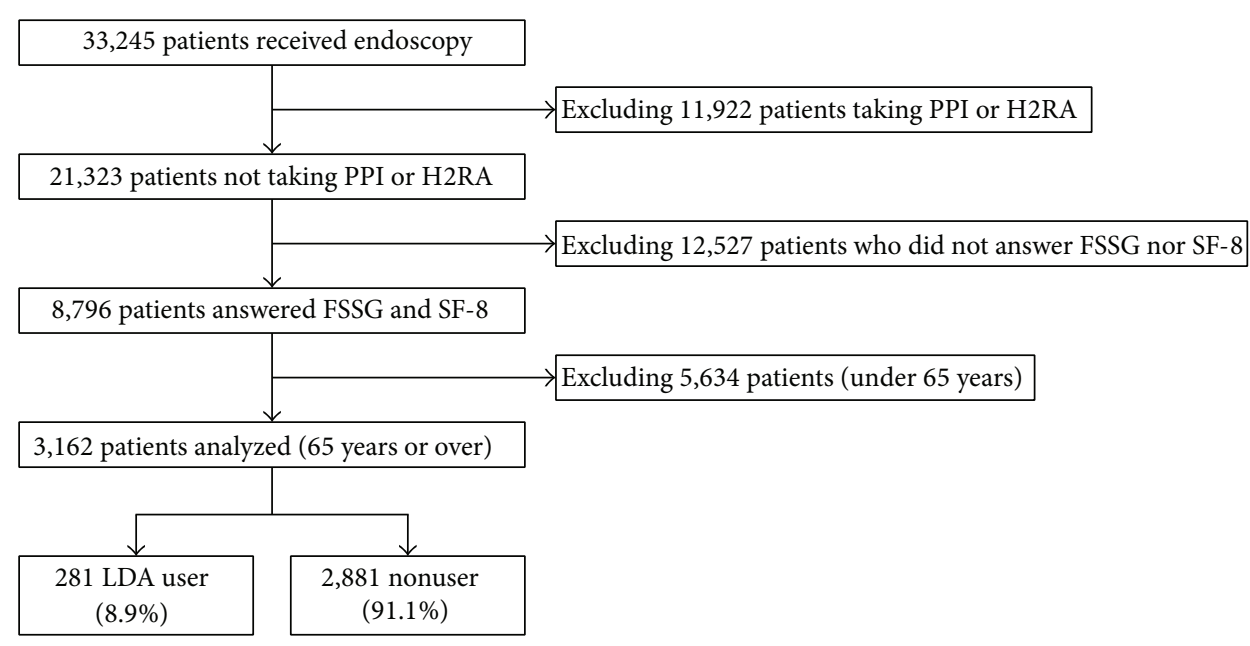

FIGURE 1: Flow chart of the study patients. PPI = proton pump inhibitor, H2RA = histamine-2 receptor antagonist, FSSG $=$ Frequency Scale for Symptoms of GERD, and SF-8 = Short-Form 8 Health Survey.

examined the relationship between taking LDA and quality of life (QOL).

In this study, we investigated the prevalence, severity, and symptoms of esophageal mucosal injury (EI), gastric mucosal injury (GI), and duodenal mucosal injury (DI) in patients taking LDA (LDA users) and not taking LDA (nonusers) to reveal the clinical features of LDA-related upper gastrointestinal mucosal injury, and to reveal the relationship between mucosal injuries among these different organs we evaluated mucosal injury among different organs in each patient.

\section{Methods}

This is a retrospective cross-sectional study performed in a single university hospital between April 2008 and December 2013. The Juntendo University Ethics Committee approved the study protocol. The performance of this study adhered to the principles of the Declaration of Helsinki for medical research involving human subjects.

Data were extracted from the records of subjects who underwent esophagogastroduodenoscopy (EGD) at our department between April 2008 and December 2013. Of the 33,245 subjects analyzed, 8,796 subjects who had filled in the Frequency Scale for Symptoms of GERD (FSSG) and ShortForm 8 Health Survey (SF-8) questionnaires were selected, after excluding subjects who took a proton pump inhibitor (PPI) or histamine-2 receptor antagonist (H2RA). Among these subjects, we focused on 3,162 subjects who were 65 years of age or over (Figure 1).

We analyzed data for subjects diagnosed endoscopically with esophageal mucosal injury (EI; Los Angeles grade A, B, $\mathrm{C}$, and D esophagitis), gastric mucosal injury (GI; gastric erosions and/or ulcers), or duodenal mucosal injury (DI; duodenal erosions and/or ulcers), individually. Gastric and duodenal mucosal injuries are based on the definition of ulcer classification which was defined by Murakami and Suzuki in 1971 [18].

We compared the prevalence, severity, and symptoms of EI, GI, and DI between LDA users and nonusers to reveal the clinical features of LDA-related upper GI mucosal injury. LDA users were defined as subjects who at the time of EGD had been regularly prescribed aspirin daily (at a dose of $81 \mathrm{mg}$ or $100 \mathrm{mg}$ ) because of chronic disease.

To assess the symptoms of patients, we employed the FSSG, which was developed for evaluation of GERD symptoms in Japanese patients and comprises the 12 most frequent symptoms $[19,20]$. This questionnaire is useful not only for objectively evaluating the therapeutic response of GERD but also potentially for patients who have GI or DI, because the FSSG items are classified into total score (TS), reflux score (RS) (questions 1, 4, 6, 7, 9, 10, and 12), and dyspepsia score (DS) (questions 2, 3, 5, 8, and 11).

To assess the QOL of patients, the SF- 8 questionnaire was used as a comprehensive scale. The SF- 8 is an alternative to the SF-36 Health Survey (SF-36) questionnaire and uses 1 question to measure each of 8 SF-36 domains. Since the SF- 8 has only 8 questions, it can be completed in 1 to 2 minutes, but it works best both for monitoring population health and for large-scale outcome studies [21]. SF-8 QOL scores were analyzed in terms of the physical component summary (PCS) and mental component summary (MCS).

Statistical analyses were performed by Mann-Whitney $U$ test. Two-sided $P$ values less than 0.05 were considered statistically significant.

\section{Results}

The study included 281 LDA users (199 men, 82 women; age range 65 to 87 years; mean age 71.7 years) and 2881 nonusers (1617 men, 1264 women; age range 65 to 93 years; mean age 72.7 years).

Figure 2 shows the prevalence of esophageal, gastric, duodenal mucosal injury and concurrent mucosal injury in 2 or more organs in LDA users and nonusers. In LDA users and nonusers, respectively, EI prevalence was 9.6\% $(n=28)$ and $10.0 \%(n=276 ; P=0.83)$, GI prevalence was $35.9 \%$ $(n=101)$ and $27.5 \%(n=792 ; P=0.0027)$, and DI prevalence was $3.3 \%(n=9)$ and $3.4 \%(n=99 ; P=0.84)$. The prevalence 


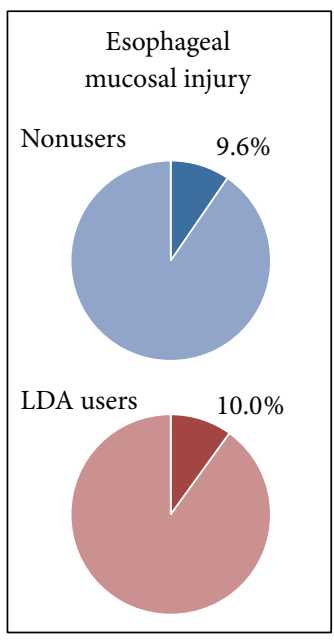

n.s.

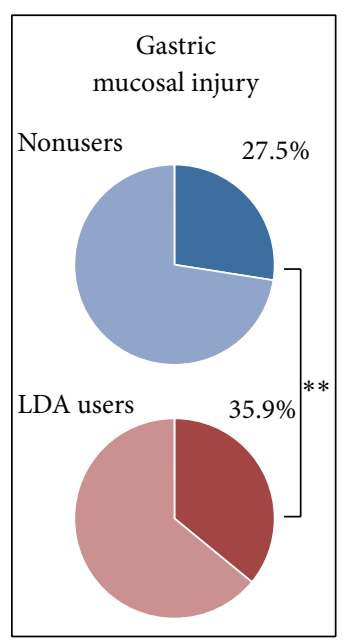

${ }^{* *} P<0.01$

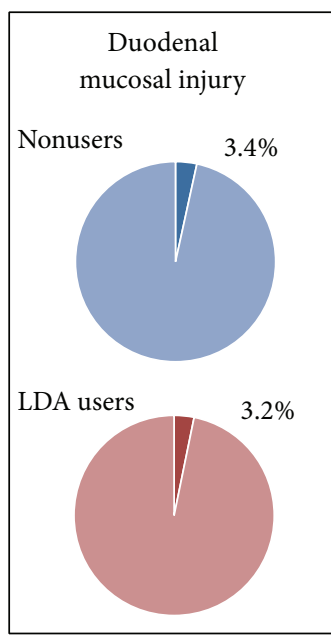

n.s.

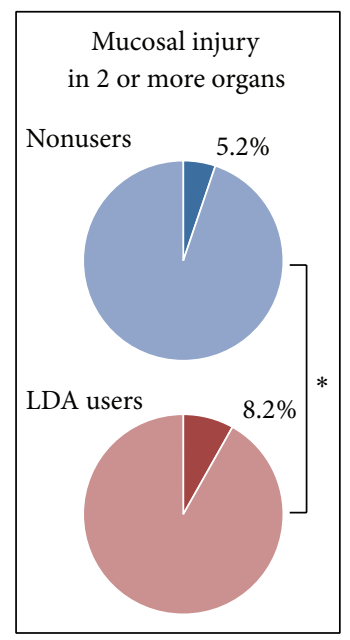

${ }^{*} P<0.05$

Figure 2: Prevalence of esophageal, gastric, and duodenal mucosal injury and concurrent mucosal injury in 2 or more organs in LDA users and nonusers. Prevalence in LDA users and nonusers was, respectively, $10.0 \%$ and $9.6 \%(P=0.83)$ for esophageal mucosal injury, $35.9 \%$ and 27.5\% $(P=0.003)$ for gastric mucosal injury, and 3.2\% and 3.4\% $(P=0.84)$ for duodenal mucosal injury. The prevalence of mucosal injury in 2 more organs was $8.2 \%$ in LDA users and $5.2 \%(P=0.04)$ in nonusers. $n$. .s. = not significant.
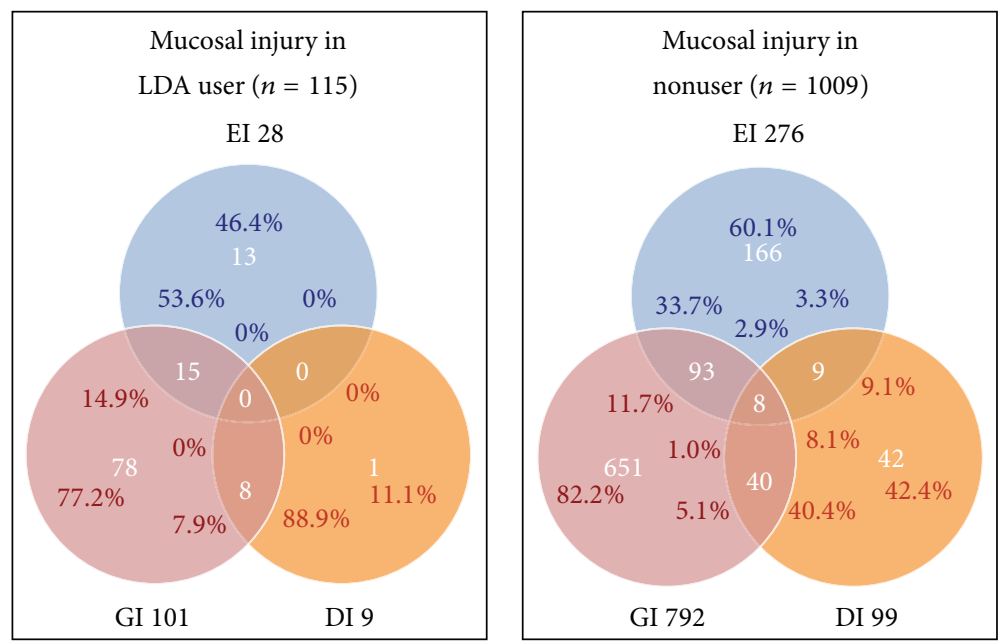

FIGURE 3: Proportions of patients with different forms of mucosal injury. In patients who had esophageal mucosal injury, the percentage with concurrent gastric mucosal injury was $53.6 \%$ for LDA users versus $33.7 \%$ for nonusers. In patients who had duodenal mucosal injury, the percentage with concurrent gastric mucosal injury was $88.9 \%$ for LDA users versus $40.4 \%$ for nonusers. EI $=$ esophageal mucosal injury, GI $=$ gastric mucosal injury, and DI = duodenal mucosal injury.

of mucosal injury in 2 or more organs concurrently (i.e., EI + $\mathrm{GI}, \mathrm{EI}+\mathrm{DI}, \mathrm{GI}+\mathrm{DI}$, or $\mathrm{EI}+\mathrm{GI}+\mathrm{DI})$ was $8.2 \%(n=23)$ in LDA users and $5.2 \%(n=150 ; P=0.036)$ in nonusers.

The proportions of patients with different forms of mucosal injury are shown in Figure 3: In patients who had esophageal mucosal injury, the percentage with concurrent gastric mucosal injury was $53.6 \%$ for LDA users versus $33.7 \%$ for nonusers. In patients who had duodenal mucosal injury, the percentage with concurrent gastric mucosal injury was 88.9\% for LDA users versus $40.4 \%$ for nonusers.

Characteristics of subjects with mucosal injury are shown in Table 1. Among subjects with GI, the proportion of men to women was higher for LDA users than nonusers.
Regarding symptoms and QOL, FSSG score of subjects with mucosal injury among LDA users and nonusers is shown in Figure 4, and SF-8 score for mucosal injury in LDA users and nonusers is shown in Figure 5. LDA users diagnosed with GI had significantly lower DS, and LDA users diagnosed with DI had significantly lower RS (Figure 4). LDA users diagnosed with EI had significantly lower PCS, and LDA users diagnosed with DI had significantly lower MCS.

\section{Discussion}

This study shows the features of LDA-related upper gastrointestinal mucosal injury in elderly LDA users and nonusers. 
TABLE 1: Characteristics of subjects with mucosal injury in individual organs.

\begin{tabular}{|c|c|c|c|c|c|c|c|c|}
\hline & \multicolumn{2}{|c|}{ Esophageal mucosal injury } & \multicolumn{2}{|c|}{ Gastric mucosal injury } & \multicolumn{2}{|c|}{ Duodenal mucosal injury } & \multicolumn{2}{|c|}{ Mucosal injury in 2 or more organs } \\
\hline & $\begin{array}{l}\text { LDA users } \\
(n=28)\end{array}$ & $\begin{array}{l}\text { Nonusers } \\
(n=276)\end{array}$ & $\begin{array}{l}\text { LDA users } \\
(n=101)\end{array}$ & $\begin{array}{c}\text { Nonusers } \\
(n=792)\end{array}$ & $\begin{array}{l}\text { LDA users } \\
\quad(n=9)\end{array}$ & $\begin{array}{c}\text { Nonusers } \\
(n=99)\end{array}$ & $\begin{array}{l}\text { LDA users } \\
(n=23)\end{array}$ & $\begin{array}{c}\text { Nonusers } \\
(n=150)\end{array}$ \\
\hline Age (year) & $72.2 \pm 5.4$ & $71.3 \pm 5.3$ & $72.1 \pm 5.2$ & $71.3 \pm 5.0$ & $71.9 \pm 5.2$ & $71.2 \pm 5.2$ & $71.4 \pm 5.1$ & $71.4 \pm 5.2$ \\
\hline $\begin{array}{l}\text { Gender (M/F) } \\
(\%)\end{array}$ & $\begin{array}{c}18 / 10 \\
(64.3 / 35.7)\end{array}$ & $\begin{array}{c}180 / 96 \\
(65.2 / 34.8)\end{array}$ & $\begin{array}{c}79 / 22 \\
(78.2 / 21.8)^{* * *}\end{array}$ & $\begin{array}{c}438 / 354 \\
(55.3 / 44.7)^{* * *}\end{array}$ & $\begin{array}{c}6 / 3 \\
(66.7 / 33.3)\end{array}$ & $\begin{array}{c}76 / 23 \\
(76.8 / 23.2)\end{array}$ & $\begin{array}{c}17 / 6 \\
(73.9 / 26.1)\end{array}$ & $\begin{array}{c}98 / 52 \\
(65.3 / 34.7)\end{array}$ \\
\hline $\operatorname{BMI}\left(\mathrm{kg} / \mathrm{m}^{2}\right)$ & $23.1 \pm 3.2$ & $23.5 \pm 4.0$ & $23.6 \pm 3.1^{* *}$ & $22.6 \pm 3.5^{* *}$ & $24.3 \pm 2.4$ & $23.0 \pm 3.0$ & $23.3 \pm 3.1$ & $24.5 \pm 3.3$ \\
\hline
\end{tabular}

${ }^{* *} P<0.01$.

${ }^{* * *} P<0.001$.

Among subjects with gastric mucosal injury, a higher proportion of men to women was evident among LDA users compared with nonusers. Data represent mean \pm SD. BMI = body mass index.

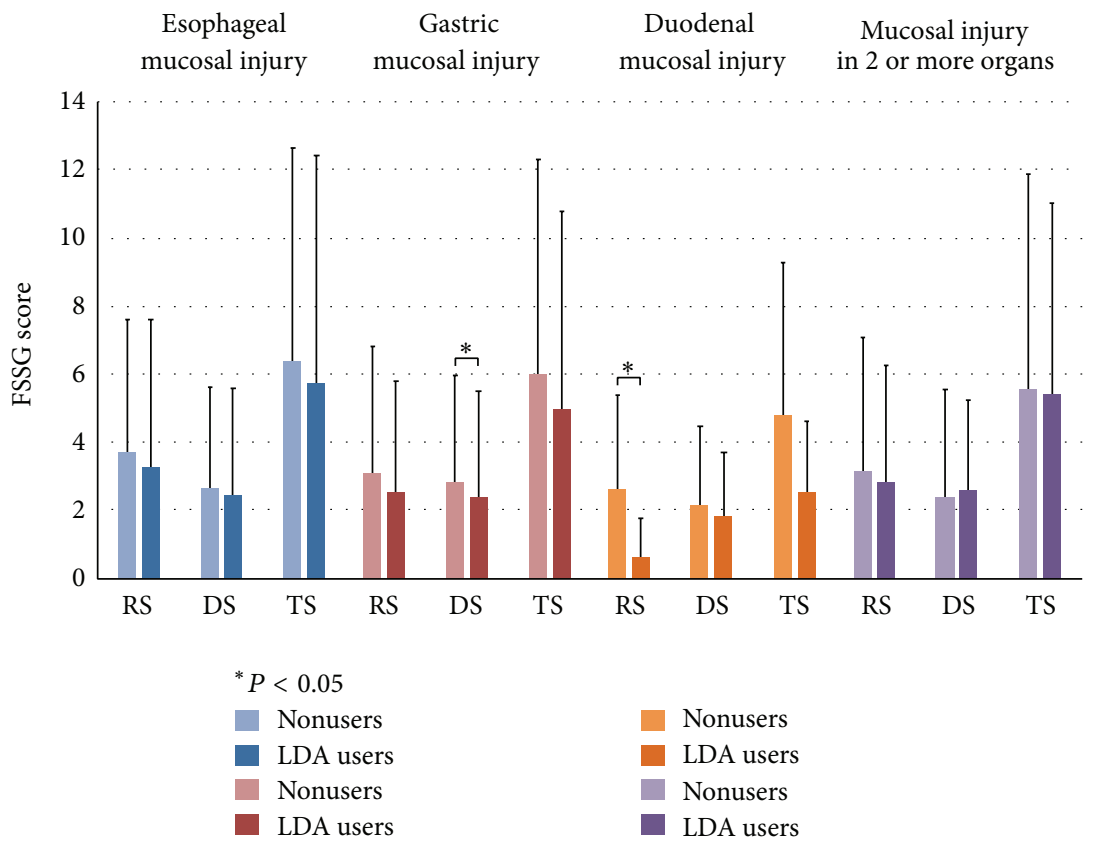

FIGURE 4: FSSG score of subjects with mucosal injury among LDA users and nonusers. Among LDA users and nonusers, respectively, the scores forTS, RS, and DS were $5.8 \pm 6.6$ and $6.4 \pm 6.3(P=0.29), 3.3 \pm 4.3$ and $3.7 \pm 3.9(P=0.17)$, and $2.5 \pm 3.1$ and $2.7 \pm 2.9(P=0.46)$ for EI; $5.0 \pm 5.8$ and $6.0 \pm 6.3(P=0.05), 2.6 \pm 3.2$ and $3.1 \pm 3.7(P=0.09)$, and $2.4 \pm 3.1$ and $2.9 \pm 3.1(P=0.04)$ for GI; $2.6 \pm 2.1$ and $4.8 \pm 4.5$ $(P=0.16), 0.7 \pm 1.1$ and $2.6 \pm 2.8(P=0.01)$, and $1.9 \pm 1.8$ and $2.2 \pm 2.3(P=0.77)$ for DI; and $5.5 \pm 6.3$ and $5.6 \pm 5.5(P=0.60), 2.9 \pm 3.9$ and $3.2 \pm 3.4(P=0.22)$, and $2.6 \pm 3.1$ and $2.4 \pm 2.6(P=0.97)$ for mucosal injury in 2 or more organs. RS $=$ reflux score, DS $=$ dysmotility score, and TS $=$ total score.

According to previous studies of peptic ulcers in patients from Western countries, the prevalence of GI in LDA users has been estimated at $65.2 \%$ [11] and 54.3\% [22], as compared with $11.8 \%$ [12] and $36.7 \%$ [23] in Japanese patients. In the present study, the prevalence of GI in LDA users was 35.9\% and was statistically significantly higher than in nonusers (27.5\%). This result was similar to findings from previous Japanese reports but lower than those from Western reports. Potential reasons for the difference include age range, race, and the rate of $H$. pylori infection [24], but the role of $H$. pylori remains unclear. In our study the proportions of $H$. pyloripositive patients among LDA users and nonusers would likely be similar; however, we did not test to confirm the presence of $H$. pylori infection. Currently, the mechanism of aspirininduced gastric mucosal damage is thought to be mediated by multiple processes involving either topical or systemic effects $[25,26]$; however, their relative contributions to aspirinrelated upper gastrointestinal mucosal injury have not yet been fully elucidated. Some reports [27, 28] have suggested that topical effects play a pivotal role in causing GI in the case of aspirin but not in the case of other NSAIDs.

The prevalence of DI in LDA users has been estimated at $24.1 \%$ [11] and $21.7 \%$ [22] in Western countries, but only $1.3 \%$ [12] and $4.7 \%$ [23] in Japan. In the present study, the prevalence of DI in LDA users was $3.2 \%$ and did not differ significantly relative to nonusers $(3.4 \%)$. The prevalence in LDA users was similar to that of other Japanese reports and consistent with the trend observed with respect to GI. The mechanisms of aspirin-induced DI differ from those of GI in several respects, because bile acid, enterobacteria, proteolytic 


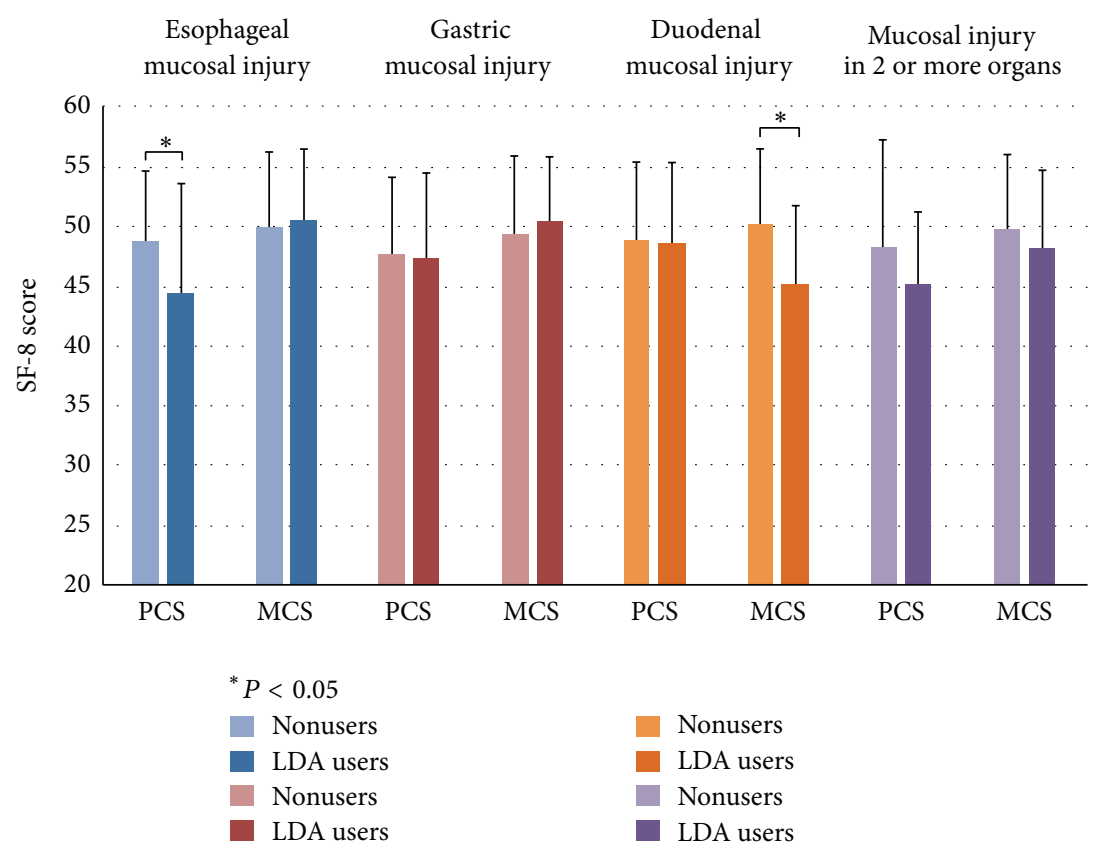

FIGURE 5: SF-8 score for mucosal injury in LDA users and nonusers. PCS and MCS among LDA users and nonusers were $44.5 \pm 9.1$ and $48.9 \pm 5.8(P=0.02)$ and $50.6 \pm 5.9$ and $50.0 \pm 6.3(P=0.73)$ for EI; $47.4 \pm 7.1$ and $47.7 \pm 6.4(P=0.75)$ and $50.5 \pm 5.4$ and $49.5 \pm 6.6(P=0.27)$ for GI; $48.8 \pm 6.7$ and $49.0 \pm 6.6(P=0.85)$ and $45.3 \pm 6.6$ and $50.3 \pm 6.3(P=0.04)$ for DI; and $45.4 \pm 9.1$ and $48.3 \pm 6.0(P=0.20)$ and $48.4 \pm 6.3$ and $49.8 \pm 6.4(P=0.28)$ for mucosal injury in 2 or more organs. PCS $=$ physical component score and MCS $=$ mental component score.

enzymes, and toxins can easily penetrate the mucosa and cause DI under conditions in which mucosal permeability is increased [29].

Opinion is divided as to whether or not aspirin is a risk factor for EI. Some multicenter, double-blind, placebocontrolled studies from Western countries have reported that LDA is a risk factor for EI [30, 31]. Conversely, a study from Japan suggested that taking LDA is not a risk factor for EI [14]. In the present study, the prevalence of EI in LDA users was $10.0 \%$ and did not differ significantly from that in nonusers (9.6\%). The prevalence in LDA users was also similar to previous reports from Japan. However, the reason for the discrepancy in the prevalence of EI in studies from Japan and Western countries remains unclear. It is thought that differences in the study populations, including age range, race, ethnicity, and the rate of $H$. pylori infection, may be responsible [32-35]. The mechanism of aspirin-induced EI remains to be fully elucidated. It is thought that inhibition by aspirin of prostaglandins derived from COX-1 weakens the esophageal mucosal defense system, as is the case with the gastric and duodenal mucosa, and allows agents such as gastric acid, pepsin, and bile salts to enter the esophageal mucosa and cause injury [36].

In the present study, we investigated subjects who were diagnosed with mucosal injury in 2 or 3 organs concurrently. Among LDA users, the prevalence of concurrent EI and GI or concurrent GI and DI was higher than in nonusers. Surprisingly, over half of patients taking LDA who had EI or DI also had concurrent GI. The reason for this result remains unknown. Previous investigators have reported that luminal acidic conditions are a necessary factor for GI and that mucosal injury is induced in an acid-dependent manner [37, 38]. We reasoned that, in such cases, inflow to the duodenum or reflux into the esophagus of excessive acidic gastric contents may subsequently cause mucosal injury.

We also investigated the relationship of LDA with symptoms and QOL. Many studies have examined the relationship between aspirin use and upper abdominal symptoms; however, it remains controversial as to whether or not the administration of aspirin can induce upper gastrointestinal symptoms [39-42]. One important factor to consider is that we focused only on elderly patients in the present study. It is known that advancing age influences visceral sensory functions and changes the degree of various symptoms, but the trend varies by type of symptoms [43-48]. However, in the present study, we found that there was no relationship between LDA and symptoms. In relation to the mechanism of impaired perception of visceral pain in elderly people, Moore and associates [49] pointed out that some age-related differences do in fact exist.

It is known that patients with peptic ulcer have lower QOL than healthy people and that patients taking aspirin experience decreased QOL. However, in our study, no significant differences were found between patients with gastric ulcer or those who did not take aspirin [50, 51]. Among patients with DI, LDA users had significantly lower MCS than nonusers. It has also been reported that GERD patients have lower QOL than do healthy people [52], but there have been no reports about QOL in GERD patients taking aspirin. In the present study, among patients with EI, PCS in LDA users was significantly lower than in nonusers. 
We have several limitations. Firstly, we might have a potential bias of patients because this is a retrospective crosssectional study, retrospective medical record review study, performed in a single university hospital. However, it may represent the common clinical site because we usually perform endoscopy not only for the patients who require detailed examinations or urgent patients, but also for the patients who just want to have a regular checkup. Secondly, only $64.1 \%$ of patients answered both FSSG and SF-8 questionnaire. It might introduce bias into the result.

In conclusion, we have demonstrated that the prevalence of gastric mucosal injury in elderly patients taking LDA was significantly higher than in nonusers. However, there was no difference in the prevalence of esophageal or duodenal mucosal injury between elderly patients who took LDA and those who did not. Furthermore, symptoms were not more prevalent in LDA users with upper gastrointestinal mucosal injury versus nonusers. These results provide important clinical information indicating that symptom-based management is not appropriate in elderly LDA users with upper gastrointestinal mucosal injuries. This is the first study to reveal the prevalence, symptoms, and QOL of upper gastrointestinal mucosal injuries in patients taking low-dose aspirin in individual organs at the same time from one same population. It is particularly unique point on this topic. We believe these results will be helpful for clinicians to manage the patients of low-dose aspirin users.

\section{Conflict of Interests}

The authors declare having no conflict of interests.

\section{References}

[1] C. Baigent, C. Sudlow, R. Collins, and R. Peto, "Collaborative meta-analysis of randomised trials of antiplatelet therapy for prevention of death, myocardial infarction, and stroke in high risk patients," British Medical Journal, vol. 324, no. 7329, pp. 7186, 2002.

[2] C. Baigent, L. Blackwell, R. Collins et al., "Aspirin in the primary and secondary prevention of vascular disease: collaborative meta-analysis of individual participant data from randomised trials," The Lancet, vol. 373, no. 9678, pp. 1849-1860, 2009.

[3] "Collaborative overview of randomised trials of antiplatelet therapy-I. prevention of death, myocardial infarction, and stroke by prolonged antiplatelet therapy in various categories of patients," The British Medical Journal, vol. 308, no. 6921, pp. 81-106, 1994.

[4] W. F. Andrawes, C. Bussy, and J. Belmin, "Prevention of cardiovascular events in elderly people," Drugs and Aging, vol. 22, no. 10, pp. 859-876, 2005.

[5] A. Lanas, M. A. Perez-Aisa, F. Feu et al., "A nationwide study of mortality associated with hospital admission due to severe gastrointestinal events and those associated with nonsteroidal antiinflammatory drug use," American Journal of Gastroenterology, vol. 100, no. 8, pp. 1685-1693, 2005.

[6] T. Hirasawa, K. Satoh, and K. Sugano, "The present state and problem of gastric ulcer caused by low-dose aspirin," Nippon Rinsho, vol. 68, no. 11, pp. 1983-1986, 2010.
[7] J. P. Kelly, D. W. Kaufman, J. M. Jurgelon, J. Sheehan, R. S. Koff, and S. Shapiro, "Risk of aspirin-associated major uppergastrointestinal bleeding with enteric-coated or buffered product," The Lancet, vol. 348, no. 9039, pp. 1413-1416, 1996.

[8] A. Lanas, E. Bajador, P. Serrano et al., "Nitrovasodilators, lowdose aspirin, other nonsteroidal antiinflammatory drugs, and the risk of upper gastrointestinal bleeding," The New England Journal of Medicine, vol. 343, no. 12, pp. 834-839, 2000.

[9] C. Capet, P. Czernichow, J.-L. Dupas et al., "Upper gastrointestinal bleeding in patients treated by low-dose aspirin," Gastroenterologie Clinique et Biologique, vol. 25, no. 3, pp. 233-238, 2001.

[10] L. Laine, "Review article: Gastrointestinal bleeding with lowdose aspirin-what's the risk?" Alimentary Pharmacology and Therapeutics, vol. 24, no. 6, pp. 897-908, 2006.

[11] N. D. Yeomans, A. I. Lanas, N. J. Talley et al., "Prevalence and incidence of gastroduodenal ulcers during treatment with vascular protective doses of aspirin," Alimentary Pharmacology and Therapeutics, vol. 22, no. 9, pp. 795-801, 2005.

[12] A. Shiotani, T. Sakakibara, Y. Yamanaka et al., "Upper gastrointestinal ulcer in Japanese patients taking low-dose aspirin," Journal of Gastroenterology, vol. 44, no. 2, pp. 126-131, 2009.

[13] S. Nakashima, S. Arai, Y. Mizuno et al., "A clinical study of Japanese patients with ulcer induced by low-dose aspirin and other non-steroidal anti-inflammatory drugs," Alimentary Pharmacology and Therapeutics, vol. 21, supplement 2, pp. 6066, 2005.

[14] T. Yamamoto, Y. Mishina, T. Ebato et al., "Prevalence of erosive esophagitis among Japanese patients taking low-dose aspirin," Journal of Gastroenterology and Hepatology, vol. 25, no. 4, pp. 792-794, 2010.

[15] J. Brun and R. Jones, "Nonsteroidal anti-inflammatory drugassociated dyspepsia: the scale of the problem," The American Journal of Medicine, vol. 110, no. 1, pp. 12S-13S, 2001.

[16] A. C. Ford, D. Forman, A. G. Bailey, A. T. R. Axon, and P. Moayyedi, "Initial poor quality of life and new onset of dyspepsia: results from a longitudinal 10-year follow-up study," Gut, vol. 56, no. 3, pp. 321-327, 2007.

[17] R. J. F. Laheij, L. G. M. van Rossum, J. B. M. J. Jansen, and F. W. A. Verheugt, "Proton-pump inhibitor therapy for acetylsalicylic acid associated upper gastrointestinal symptoms: a randomized placebo-controlled trial," Alimentary Pharmacology and Therapeutics, vol. 18, no. 1, pp. 109-115, 2003.

[18] T. Murakami and T. Suzuki, Everithing of the Stomach and Duodenum, Nanko-do, Tokyo, Japan, 1971.

[19] N. D. Yeomans and J. Naesdal, "Systematic review: ulcer definition in NSAID ulcer prevention trials," Alimentary Pharmacology and Therapeutics, vol. 27, no. 6, pp. 465-472, 2008.

[20] M. Kusano, Y. Shimoyama, S. Sugimoto et al., "Development and evaluation of FSSG: frequency scale for the symptoms of GERD," Journal of Gastroenterology, vol. 39, no. 9, pp. 888-891, 2004.

[21] J. Ware, M. Kosinski, J. Dewey, and B. Gandek, How to Score and Interpret Single-Item Health Status Measures: A Manual for Users of the SF-8 Health Survey, QualyMetric, Lincoln, RI, USA, 2001.

[22] Y. Niv, A. Battler, G. Abuksis, E. Gal, B. Sapoznikov, and A. Vilkin, "Endoscopy in asymptomatic minidose aspirin consumers," Digestive Diseases and Sciences, vol. 50, no. 1, pp. 78-80, 2005.

[23] A. Tamura, K. Murakami, and J. Kadota, "Prevalence and independent factors for gastroduodenal ulcers/erosions in asymptomatic patients taking low-dose aspirin and gastroprotective 
agents: the OITA-GF study," QJM: An International Journal of Medicine, vol. 104, no. 2, pp. 133-139, 2011.

[24] F. K. L. Chan, "Should we eradicate Helicobaster pylori infection in patients receiving nonsteroidal anti-inflammatory drugs or low-dose aspirin?" Chinese Journal of Digestive Diseases, vol. 6, no. 1, pp. 1-5, 2005.

[25] C. Musumba, D. M. Pritchard, and M. Pirmohamed, "Review article: cellular and molecular mechanisms of NSAID-induced peptic ulcers," Alimentary Pharmacology \& Therapeutics, vol. 30, no. 6, pp. 517-531, 2009.

[26] Y. Naito and T. Yoshikawa, "Pathogenesis of aspirin-induced gastric mucosal injury," Nippon Rinsho, vol. 68, no. 11, pp. 20072014, 2010.

[27] Y. Mashita, M. Taniguchi, A. Yokota, A. Tanaka, and K. Takeuchi, "Oral but not parenteral aspirin upregulates COX2 expression in rat stomachs: a relationship between COX-2 expression and PG deficiency," Digestion, vol. 73, no. 2-3, pp. 124-132, 2006.

[28] B. Cryer, D. L. Bhatt, F. L. Lanza, J.-F. Dong, L. M. Lichtenberger, and U. K. Marathi, "Low-dose aspirin-induced ulceration is attenuated by aspirin-phosphatidylcholine: a randomized clinical trial," The American Journal of Gastroenterology, vol. 106, no. 2, pp. 272-277, 2011.

[29] I. Bjarnason, J. Hayllar, A. J. MacPherson, and A. S. Russell, "Side effects of nonsteroidal anti-inflammatory drugs on the small and large intestine in humans," Gastroenterology, vol. 104, no. 6, pp. 1832-1847, 1993.

[30] A. S. Taha, C. McCloskey, R. Prasad, and V. Bezlyak, "Famotidine for the prevention of peptic ulcers and oesophagitis in patients taking low-dose aspirin (FAMOUS): a phase III, randomised, double-blind, placebo-controlled trial," The Lancet, vol. 374, no. 9684, pp. 119-125, 2009.

[31] N. Yeomans, A. Lanas, J. Labenz et al., "Efficacy of esomeprazole (20 mg once daily) for reducing the risk of gastroduodenal ulcers associated with continuous use of low-dose aspirin," The American Journal of Gastroenterology, vol. 103, no. 10, pp. 24652473, 2008.

[32] B. Peleteiro, A. Bastos, A. Ferro, and N. Lunet, "Prevalence of Helicobacter pylori infection worldwide: a systematic review of studies with national coverage," Digestive Diseases and Sciences, vol. 59, no. 8, pp. 1698-170, 2014.

[33] H. M. Malaty, L. Engstrand, N. L. Pedersen, and D. Y. Graham, "Helicobacter pylori infection: genetic and environmental influences. A study of twins," Annals of Internal Medicine, vol. 120, no. 12, pp. 982-986, 1994.

[34] H. M. Malaty, D. Y. Graham, I. Isaksson, L. Engstrand, and N. L. Pedersen, "Are genetic influences on peptic ulcer dependent or independent of genetic influences for Helicobacter pylori infection?" Archives of Internal Medicine, vol. 160, no. 1, pp. 105$109,2000$.

[35] K. Haruma, T. Kamada, H. Kawaguchi et al., "Effect of age and Helicobacter pylori infection on gastric acid secretion," Journal of Gastroenterology and Hepatology, vol. 15, no. 3, pp. 277-283, 2000.

[36] A. Lanas, P. Jiménez, A. Ferrández et al., "Selective COX-2 inhibition is associated with decreased mucosal damage induced by acid and pepsin in rabbit esophagitis," Inflammation, vol. 27, no. 1, pp. 21-29, 2003.

[37] W. P. Ritchie Jr., "Acute gastric mucosal damage induced by bile salts, acid and ischemia," Gastroenterology, vol. 68, no. 4, pp. 699-707, 1975.
[38] P. H. Rowe, M. J. Starlinger, E. Kasdon, M. J. Hollands, and W. Silen, "Parenteral aspirin and sodium salicylate are equally injurious to the rat gastric mucosa," Gastroenterology, vol. 93, no. 4, pp. 863-871, 1987.

[39] S. Nandurkar, N. J. Talley, H. Xia, H. Mitchell, S. Hazel, and M. Jones, "Dyspepsia in the community is linked to smoking and aspirin use but not to Helicobacter pylori infection," Archives of Internal Medicine, vol. 158, no. 13, pp. 1427-1433, 1998.

[40] A. K. Tuteja, N. J. Talley, S. K. Joos, K. G. Tolman, and D. H. Hickam, "Abdominal bloating in employed adults: prevalence, risk factors, and association with other bowel disorders," The American Journal of Gastroenterology, vol. 103, no. 5, pp. 12411248, 2008.

[41] C. Belanger, J. E. Buring, N. Cook et al., "Final report on the aspirin component of the ongoing physicians' health study," New England Journal of Medicine, vol. 321, no. 3, pp. 129-135, 1989.

[42] J. Jaspers Focks, M. M. Tielemans, L. G. M. van Rossum et al., "Gastrointestinal symptoms in low-dose aspirin users: a comparison between plain and buffered aspirin," Netherlands Heart Journal, vol. 22, no. 3, pp. 107-112, 2014.

[43] L. Kay, T. Jorgensen, and K. H. Jensen, "Epidemiology of abdominal symptoms in a random population: prevalence, incidence, and natural history," European Journal of Epidemiology, vol. 10, no. 5, pp. 559-566, 1994.

[44] L. Agréus, K. Svärdsudd, N. J. Talley, M. P. Jones, and G. Tibblin, "Natural history of gastroesophageal reflux disease and functional abdominal disorders: a population-based study," The American Journal of Gastroenterology, vol. 96, no. 10, pp. 29052914, 2001.

[45] H. Lasch, D. O. Castell, and J. A. Castell, "Evidence for diminished visceral pain with aging: studies using graded intraesophageal balloon distension," The American Journal of Physiology-Gastrointestinal and Liver Physiology, vol. 272, no. 1, pp. G1-G3, 1997.

[46] M. Gururatsakul, R. H. Holloway, B. Adam, T. Liebregts, N. J. Talley, and G. J. Holtmann, "The ageing gut: diminished symptom response to a standardized nutrient stimulus," Neurogastroenterology and Motility, vol. 22, no. 3, pp. 246-e77, 2010.

[47] C. K. Redmond, J. Gustin, and E. Kamon, "Long term mortality experience of steelworkers. VIII. Mortality patterns of open hearth steelworkers (a preliminary report)," Journal of Occupational Medicine, vol. 17, no. 1, pp. 40-43, 1975.

[48] D. A. Johnson and M. B. Fennerty, "Heartburn severity underestimates erosive esophagitis severity in elderly patients with gastroesophageal reflux disease," Gastroenterology, vol. 126, no. 3, pp. 660-664, 2004.

[49] A. R. Moore and D. Clinch, "Underlying mechanisms of impaired visceral pain perception in older people," Journal of the American Geriatrics Society, vol. 52, no. 1, pp. 132-136, 2004.

[50] K. Tominaga, K. Higuchi, T. Iketani et al., "Comparison of gastrointestinal symptoms and psychological factors of functional dyspepsia to peptic ulcer or panic disorder patients," Inflammopharmacology, vol. 15, no. 2, pp. 84-89, 2007.

[51] M. Watanabe, T. Kawai, Y. Takata, and A. Yamashina, "Gastric mucosal damage evaluated by transnasal endoscopy and QOL assessments in ischemic heart disease patients receiving lowdose aspirin," Internal Medicine, vol. 50, no. 6, pp. 539-544, 2011.

[52] J. Tack, A. Becher, C. Mulligan, and D. A. Johnson, "Systematic review: the burden of disruptive gastro-oesophageal reflux disease on health-related quality of life," Alimentary Pharmacology and Therapeutics, vol. 35, no. 11, pp. 1257-1266, 2012. 


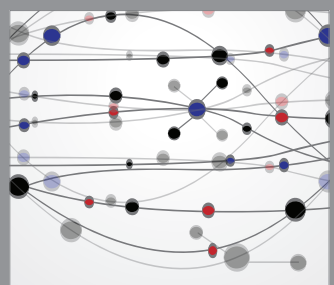

The Scientific World Journal
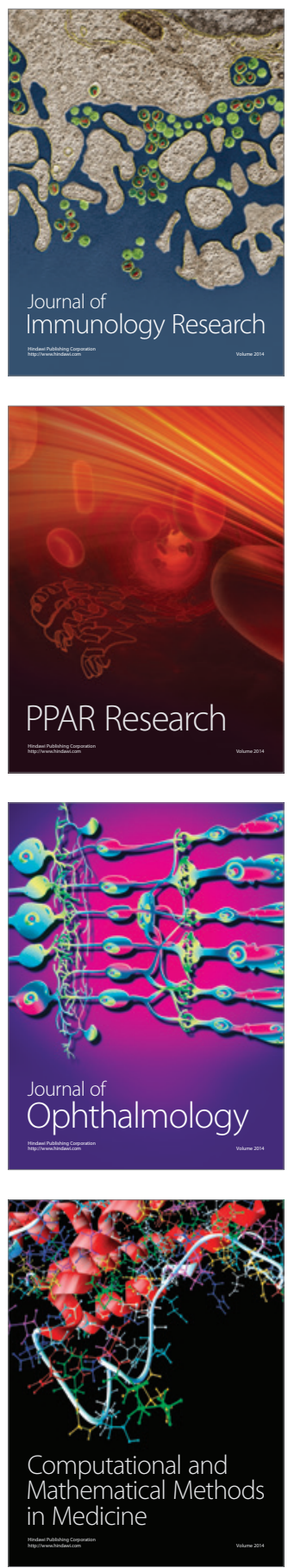

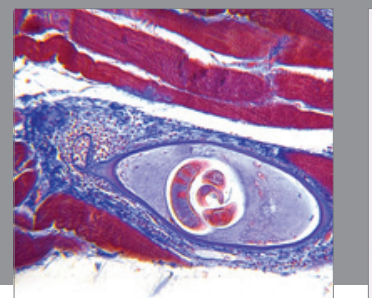

Gastroenterology

Research and Practice
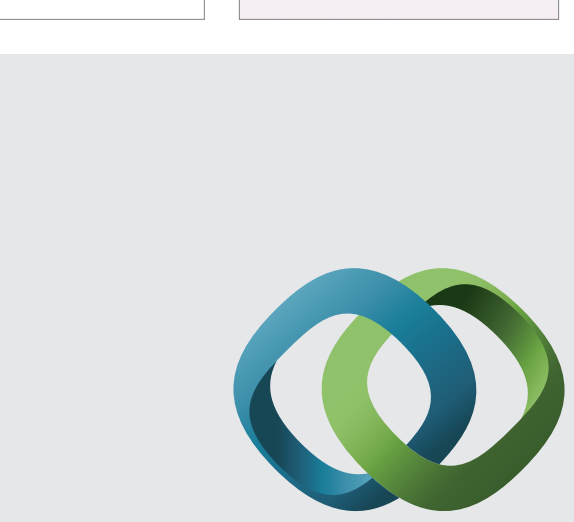

\section{Hindawi}

Submit your manuscripts at

http://www.hindawi.com
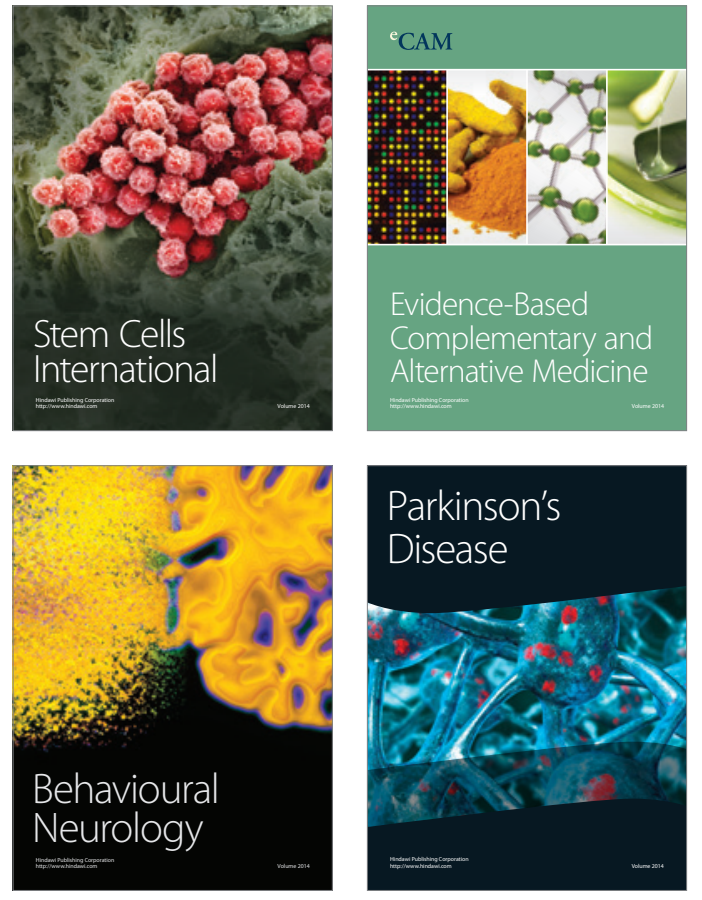
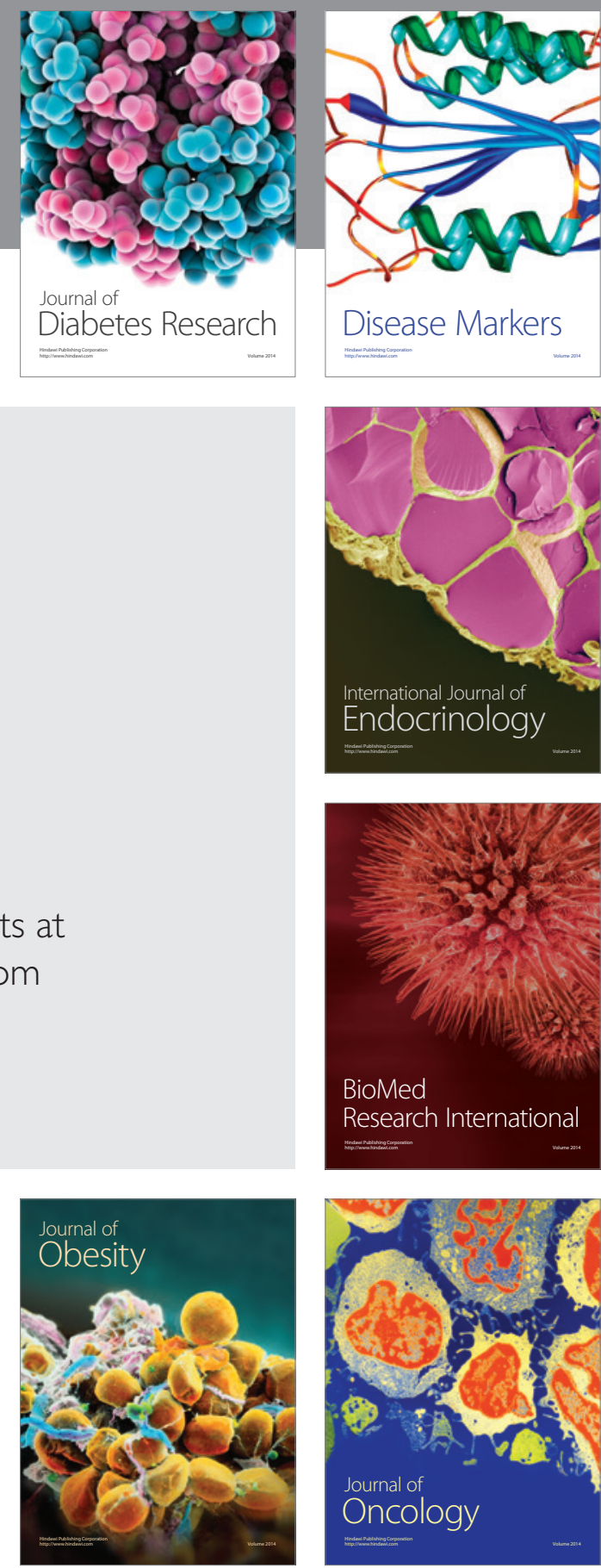

Disease Markers
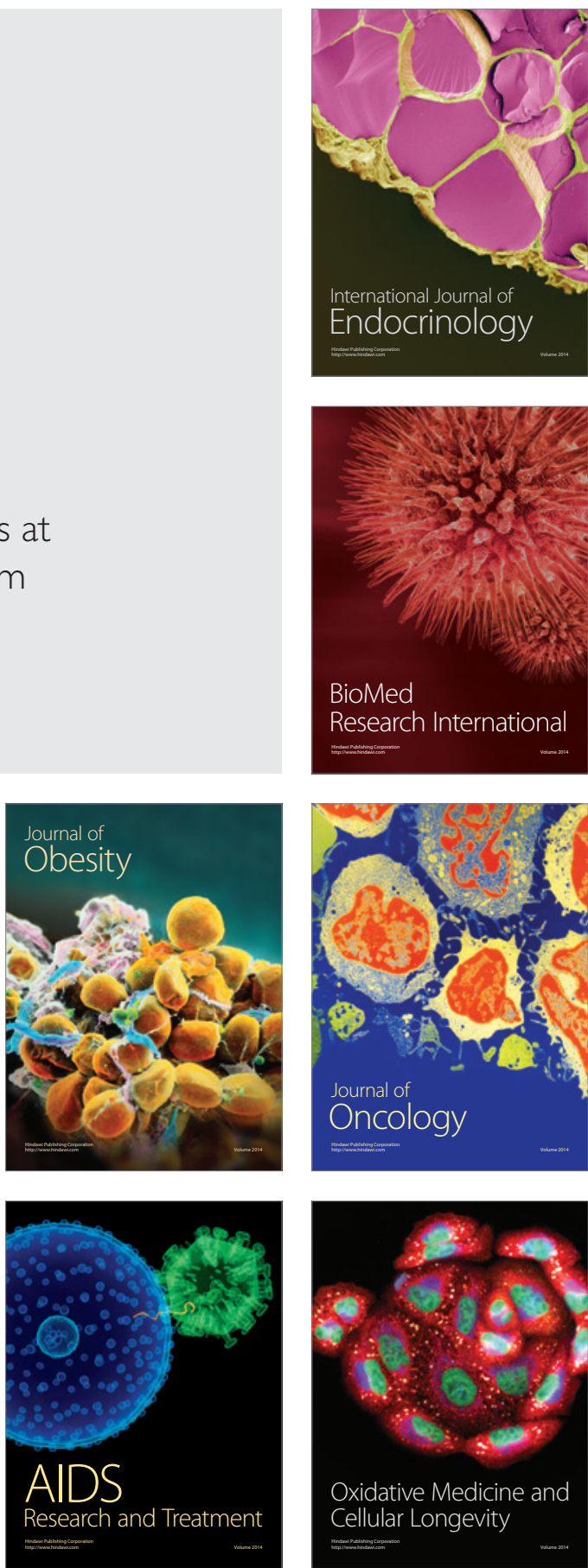